Bol. Acad. peru. leng. 58. 2014 (61-76)

\title{
LA POÉTICA DEL GRAN DISPARATE Y EL RITMO PEDAL DE TRILCE
}

\section{LA POÉTIQUE DU GRAND ABSURDE ET LE RYTHME MARCHEUR DE TRILCE}

\section{POETICS OF GREAT ABSURDITY AND THE PEDAL RHYTHM OF TRILCE O}

\author{
Óscar Coello \\ Universidad Nacional Mayor de San Marcos
}

\section{Resumen:}

Este artículo muestra algunos apuntes acerca de la aventura poética de Trilce, desde el plano general de la concepción del trabajo artístico hasta el plano puntual de la elaboración versal, donde el ritmo de cadencia pedal actúa como eje que vertebra los sentidos y la sintaxis extraviada del discurso.

\section{Résumé:}

Cet article contient certaines observations sur l'aventure poétique de Trilce, du plan général de la conception du travail artistique au plan ponctuel de l'élaboration du vers, où le rythme à la cadence marcheuse agit comme un axe structurant les sens et la syntaxe égarée du discours. 


\section{Abstract:}

This article shows some notes about the poetic adventure of Trilce, from the general level of the conception of the artwork to the specific level of the verse production, where the rhythm of pedal cadence acts as axis that structures the senses with the lost syntax of speech.

Palabras clave: Poética, ritmo, Vallejo, Trilce.

Mots clés: Poétique, rythme, Vallejo, Trilce.

Key words: Poetics, rhythm, Vallejo, Trilce.

Fecha de recepción: $\quad$ 26/09/2014

Fecha de aceptación: $\quad$ 20/11/2014

\section{Acerca de la poética}

Demás está decir que en las ciencias humanas las fronteras terminológicas no tienen tratados de límites y, menos, tratados perpetuos, de modo que no todos entienden las cosas de una misma manera. A veces, los términos se confunden; por ello, estamos obligados a un ejercicio de permanente alerta en nuestros esfuerzos por tratar de entendernos. Hay un sentido general en el que se habla de poética, vale decir, el que la acepta como un dominio de la ciencia aplicado a un sector de la realidad que es la poesía. Hay sentidos menos estrictos con los que se emplea este término, como aquel que plantea que la poética aborda el estudio de una modalidad especial del discurso verbal, que es el discurso artístico; y otros muchísimo menos estrictos o, mejor diré, más amplios que la definen ya no como posibilidad científica, sino como una simple reflexión. Citaré a un recordado profesor sanmarquino de Teoría Literaria, Edmundo Bendezú; él decía entender «... la Poética no como una ciencia, sino como un 
conjunto de respuestas a las interrogantes que el hombre se ha planteado sobre ese fenómeno extraordinario que se llama poesía» ${ }^{1}$.

En este artículo voy a emplearun sentido todavía más ajustado; ese que - acercándose al trabajo creador o a la Poiesis del artista en un poema o un libro determinado- entiende la Poética como el Arte Poética, es decir, como el arte de concebir y de forjar el poema. Ello nos permitirá hacer razonamientos acerca del planteamiento, por parte del artista, de una propuesta formal para ejercitar un quehacer poético en particular. En este caso concreto, el de la poética que ilustra el libro cenital del artista César Vallejo. Por lo demás, este es el sentido de poética que suele emplear la crítica contemporánea para indagar acerca de los supuestos que sostienen las columnatas del poema o los supuestos del libro de poemas de un determinado autor.

\section{En busca de la poética de Trilce}

Como sabemos, no hay en todo Trilce ningún poema que deliberadamente ni implícitamente se titule Arte Poética o Ars Poética o Poiesis. Es decir, desde el poema «I» hasta el poema «LXXVII», con el que se cierra el libro, no hay un solo texto en donde esté explícita, o al menos clara, la voluntad del artista por dejar sentadas las estipulaciones dentro de las cuales se debe entender su abordaje del trabajo versal.

Pero si el poeta de Trilce no ha querido proclamar en algún poema del libro su derrotero artístico, eso no quiere decir que la crítica del pasado siglo no se haya propuesto conjeturar tal entender de algún modo.

Quisiera proponer dos casos ejemplares de estas búsquedas de la poética de Trilce; de suyo diferentes. Uno, el que propone el poema «I» de Trilce como la poética del libro; otro que se va al otro extremo y propone el poema «LXXVII», con el que termina el libro, como la poética; después, propondré mi modo de ver el problema, o mejor aún,

1 Bendezú, Edmundo. El delirio de los coribantes. (Estudios sobre poética). Lima: P. L. Villanueva, 1981, p. 9. 
mi propuesta para encarar la conjetura de la poética de Trilce. Así, solo como una propuesta para encarar el problema.

\section{El poema «I» como poética}

El vallejista Keith McDuffie, de la Universidad de Montana, en un recordado artículo aparecido en la Revista Iberoamericana, N. ${ }^{\circ} 71$, allá por 1970 , luego de un minucioso análisis textual del poema «I», donde sostenía que «bulla significa el caos de la vida» ${ }^{2}$ y que: «De tal caos el poeta quiere sacar un orden existencial, las islas, que en primer plano son las islas guaneras sobre la costa peruana, pero que en otro plano simbólico son los recuerdos o memorias de una existencia más ordenada y ya pasada» ${ }^{3}$, etc., concluye que: «Se trata nada menos que de la poética fundamental de Trilce, el planteamiento de una nueva dimensión existencial actual y la proyección del poeta hacia un plano ideal» ${ }^{4}$.

Como es de ver aquí, los parámetros de la conjetura son puramente impresionistas; todas estas son solo expresiones que cifran las creencias personales del crítico.

Por este solo tipo de razonamientos (o, mejor, debo hablar de 'impresiones' ante el texto) no nos queda claro que el poema «I» deba ser aceptado como la poética o ars poética de Vallejo para este libro. Resulta obvio que las citas leídas - u otras similares que no he leído, y que conforman el aparato probatorio de la tesis de que este poema es la poética de Trilce - resultan ser solo afirmaciones personales de Keith McDuffie; $y$, por tanto, pasibles del disenso. Justo es decir que era el tipo de discurso crítico de la época y aceptado con el mejor de los respetos. Respeto, por supuesto, que es el mismo que guardo al exhibir este artículo y sus afanes.

Acaso podríamos señalar también que, tal vez, si el poeta de Trilce nos hubiera dejado textos metapoéticos — a la manera de los que hicieron

2 McDuffie, Keith: “"Trilce I” y la función de la palabra en la poética de César Vallejo». En: Revista Iberoamericana. Pittsburgh, Volumen XXXVI, N. ${ }^{\circ} 71,1970$, p. 196.

3 Ídem.

4 Ibíd., p. 203. 
Dante o Juan de la Cruz o Juan de Mena- que explicaran sus poemas, y allí se precisara el significado de bulla o de islas, tal cual lo quiere Keith McDuffie, la conjetura sería más propicia.

\section{El poema «LXXVII» como poética}

El segundo ejemplo que tomo como paradigma de estos afanes por conjeturar el arte poética de Trilce, en el pasado siglo, es uno que propone el poema «LXXVII» como "la poética" del libro. El poema «LXXVII», como sabemos, es el último de Trilce. Y la propuesta la formuló Julio Ortega por los mismos años del juicio de Keith McDuffie, que acabamos de ver. En efecto, en su libro Figuración de la persona, Barcelona, EDHASA, año de 1971, capítulo "Trilce, cuestionamientos de la persona», p. 81, dice: «Creo que el poema LXXVII puede ser leído como poética central de Trilce». Mas toda la argumentación probatoria tampoco se cimienta en un análisis textual amparado por ninguna metodología conocida, sino que también se levanta sobre el «creo», de la cita leída, y sobre un frágil «parece suponer», al inicio de la argumentación. En efecto, la premisa fundamental de la que parte dice: «El tú implícito al que se dirige el poema parece suponer el acto poético» ${ }^{5}$. Y sigue, «a la poesía, que es una lluvia dentro de una tempestad $»^{6}$. A partir de allí, obran las disquisiciones personales de Ortega como intérprete de los sentidos profundos de Trilce, por lo que podemos o no, estar de acuerdo con él.

\section{Texto y contexto en la búsqueda de la poética de Trilce}

He dicho que no hay en todo Trilce ningún poema que deliberadamente ni implícitamente se titule Arte Poética o Ars Poética o Poiesis. En consecuencia, planteo aquí que toda conjetura de su poética se tendrá que hacer no privilegiando ningún poema en especial, sino recorriendo todos los textos del libro; y sin desatender objetivamente nada de lo que se ha publicado como poema en el propio libro o fuera de él. Me refiero a los

5 Ortega, Julio: Figuración de la persona. Barcelona: Edhasa, 1971, p. 82.

6 Ídem. 
poemas publicados en el libro, en 1922 o antes de esa fecha en forma aislada, por el propio poeta.

Examinemos los textos. Trilce está en verso libre, pero no solo está en verso libre. Hay poemas que no abdican de la métrica o la rima; y en ningún poema de los 77 del libro se abdica del ritmo. Trilce es un prodigio de versos innovadores, pero también tiene un fuerte fondo de versos claros y alberga estrofas tradicionales. La cuestión es ponernos a contar objetivamente y establecer los porcentajes para arribar a conclusiones que no satisfagan nuestros prejuicios, sino que respondan a nuestra razón serena. Más aún es un dato objetivo que se conservan versiones publicadas en forma aislada de poemas cenitales — antes de la edición del libro, en 1922 - que nos pueden dar luces sobre la configuración de los textos definitivos.

Felizmente, ya están hechos muchos conteos y las herramientas de análisis empleadas son de lo más confiables y verificables, y dejan fuera toda subjetividad. Se hicieron en la década siguiente a la de los citados estudios anteriores. Voy a referirme a uno de ellos solamente, para evitar prolijidad, como decían los antiguos.

Por ejemplo, mostraré cómo por la década de los ochenta, y desde las canteras de la estilística y del estructuralismo, hay trabajos que ofrecen mediciones solventes. Miremos aquí el minucioso trabajo de la estudiosa Irene Vegas García en Trilce, estructura de un nuevo lenguaje. Lima, Pontificia Universidad Católica del Perú, Fondo Editorial, del año de 1982 - y con ello le rindo un homenaje a la maestra a la que seguí en un memorable seminario sobre Vallejo, justo por esos años ochenta, en que me encontraba como estudiante del magíster de Literaturas Hispánicas en la Pontificia Universidad Católica del Perú—. Irene Vegas, hija de un intelectual piurano y sanmarquino, don Ricardo Vegas García, buen amigo de Vallejo, recibió de su padre un interminable amor por la poesía del artista. Ella nos mostró aquella vez, en su citado libro, algunos logros obtenidos desde marcas, repito, verificables en el texto y que exhibió como indicios, así, como meros indicios, de una inclinación teórica de la voz poética hacia la configuración artística de Trilce. Y ya no solo eli- 
giendo voluntariosamente el poema «I» o el «LXXVII» como «poéticas» del libro, sino eligiendo la opción de rastrear por todo el libro marcas textuales que amparen la conjetura. Admito también que es cierto que Irene Vegas nunca usó el término poética para mostrar sus logros.

Irene Vegas encontró que el libro - escrito preferentemente en verso libre (pero no solo en verso libre) y dentro de los parámetros de ese verso sin metro ni rima- se complacía en propiciar el establecimiento de un ritmo insólito con la flagrante desviación de la pausa versal. Así, Irene Vegas informó que había un $35.98 \%$ de versos que terminaban en pausas no concordadas, es decir, de aquellas que no mantienen el paralelismo fonosemántico y que servían para instaurar el desconcierto rítmico ${ }^{7}$. Citaré algunos ejemplos: «Lado al lado al destino y llora», «Bomba aburrida del cuartel achica», "Y he aquí se me cae la baba, soy», "Cuánto se aceita en codos», etc. También señaló que, en cuanto al nivel semántico, Trilce era un prodigio de predicados impertinentes: de los 2,605 versos que tiene el poemario, un $59.31 \%$, es decir, 1545 , están constituidos por atributos desviantes ${ }^{8}$, donde los casos extremos se pueden ejemplificar por los versos: «el guano, la simple calabrinatesórea», «Julio estaba entonces de nueve»; «sacando la lengua a las más mudas equis», etc.

No es mi propósito, por cierto, referir aquí la totalidad del campo de mediciones que Irene Vegas García determinó en 1982; y, menos, dar cuenta de todas las otras comprobaciones que, como las de Irene Vegas, han realizado otros estudiosos que han partido de los propios textos de Trilce para someterlos a un análisis puntual que se aparte del decir subjetivo. Solo quisiera resaltar aquí que es desde este tipo de mediciones por donde podremos caminar hacia la conjetura cierta que nos explique, con mayor grado de verosimilitud, las grandes orientaciones del trabajo creador vallejiano, aún aureolado de misterio. Solo con recuentos como el mostrado, estaremos en condiciones de levantar afirmaciones con visos de verdad en busca de la naturaleza del planteo poético vallejiano.

\footnotetext{
7 Vegas García, Irene: Trilce, estructura de un nuevo lenguaje. Lima: Pontificia Universidad Católica del Perú - Fondo Editorial, 1981, p. 20 y ss.

8 Ibíd., p. 36.
} 
Pero no todo Trilce es poesía transracional. Si se tratara de puntualizar o precisar hasta aquí nuestros apuntes, diremos que por trabajos como el referido podemos sacar las cuentas claras de cómo Vallejo se define preferente - pero no excluyentemente- hacia la creación de un discurso insólito, libérrimo. Diría yo que queda bien medida (o cuantificada) la deliberada propuesta vallejiana para —en un buen número de casos, pero nunca olvidemos que no son el $100 \%$, sino mucho menosdecir las cosas como no se dicen, para asomarse a los bordes del abismo de la expresión ininteligible per se, para separar la dicción poética de la dicción diaria, para interponer un puente de silencio entre lo que el mundo dice y lo que el poeta habla o no habla en su poesía; en fin, que la voz poética construye con su discurso un discurso estético de ruptura, pero sin acabar por salirse totalmente de los cauces del sentido, tal como lo muestran — repito - los porcentajes. Queda claro que hacer poesía, para Vallejo, era hablar solo en un «lenguaje extraño y obstruyente» (así definía Shklovski, un formalista ruso, la poesía, por la misma época de Vallejo), pero que no llega a ningún extremismo de vaciar de sentido todo el discurso; es más, en Trilce encontramos que hay poemas - como el famoso «LXV», «Madre, me voy mañana a Santiago»— que son de una clarísima inteligencia versal y verbal.

Ahora, quisiera completar el campo de las mediciones textuales con las noticias contextuales. Sabemos que en Trilce, Vallejo se adhiere a una nueva concepción del arte. Sabemos que el poeta, en Trilce, busca situarse dentro de ese movimiento intelectual insurrecto que se descreía de los fundamentos mismos de la civilización, y de los más firmes supuestos establecidos por nuestro saber temporal; concretamente, de la Razón y de la Lógica. Estas habían conducido a la raza humana hacia su autodestrucción en la Primera Guerra Mundial, ya que se habían mostrado impertérritas en el perfeccionamiento de una maquinaria guerrera feroz, donde los nuevos inventos (como la aviación) no habían venido sino a hacer más audaces los bombardeos de ciudades, y más certeras las matanzas. Un mundo donde los nuevos artefactos (como la rudimentaria máquina fotográfica) daban lecciones de mímesis y de simetría a la mano y al ojo del hombre. La postura estética del poeta, pues, lo sabemos, estaba cantada por la vanguardia 
internacional que tentó sin temores la posible salida ilógica o la buscó por el lado no previsto del absurdo. Así, en este contexto, el planteo artístico del poema bien podía admitir la opción irracional hasta con alguna naturalidad; y, así también, la poesía no vacilaría en proponer sin más el absurdo como salida. El libro deja huellas claras de que el poeta estaba en este sendero. Insisto, aunque tampoco discurría por él ciega y obcecadamente.

Ahora regreso al texto poético, pues debo citar algunos versos que amparen lo que acabo de decir. Veamos estos del poema «LXXIII»:
Absurdo, solo tú eres puro.
Absurdo, este exceso solo ante ti se suda de dorado placer.

En el mismo poema concluye:

$$
\begin{aligned}
& \text { Tengo pues derecho } \\
& {[\ldots] \text { a meter la pata }[\ldots]}
\end{aligned}
$$

Pero no solo del poema «LXXIII» podemos sacar citas parecidas a estas; también las hay en otros poemas. Sin pretender agotar el repertorio, cito también el poema «XXXVI», ya mencionado, donde hay versos que son todo un deslinde estético:

Rehusad, y vosotros, a posar las plantas

en la seguridad dupla de la Armonía.

Rehusad la simetría a buen seguro.

Es decir — si no la simetría, si no la armonía- es posible convivir con el disparate. En claros versos emblemáticos del libro la voz poética proclama:

Pugnamos ensartarnos por un ojo de aguja [...] 
En otro:

iCeded al nuevo impar

potente de orfandad!

Y en otro:

¡Hembra se continúa el macho [...]

En el poema «XLV», el artista resume así su postura estética:

Y si así diéramos las narices

en el absurdo,

nos cubriremos con el oro de no tener nada, y empollaremos el ala aún no nacida $[\ldots]$

de esta ala huérfana del día, que a fuerza de ser una ya no es ala.

El discurso absurdo, ilógico, no se explica, no se traduce. Sus profundos sentidos los descodifica el alma en sus adentros. De este modo, Trilce es un libro profundo. En muchos momentos, es pura poesía inexplicable, intraducible, pero hondamente decidora. En otros, como he dicho, el discurso es claro como el agua: «Se acabó el extraño, con quien tarde / la noche, regresabas parla y parla» o el citado «Madre, me voy mañana a Santiago, / a mojarme en tu bendición y en tu llanto».

\section{El ritmo de cadencia pedal enTrilce}

Ahora voy a trabajar el rasgo distintivo que sostiene el poema cuando el poeta acude a la sintaxis del absurdo, o a la semántica inaprensible que a veces enfrentamos en Trilce. Este factor es el ritmo. Por ritmo voy a entender, en sentido general, toda reiteración en el discurso. En el caso de la poesía, Rafael de Balbín ${ }^{9}$ concluye que el ritmo se consigue, en primer lugar, por la meditada distribución de los golpes acentuales en

9 Balbín, Rafael de: Sistema de rítmica castellana. Madrid, Gredos, 1975, 3. a ed. 
la frase versal, y así se le llama ritmo de intensidad. El ritmo también se consigue por la longitud del verso o ritmo de cantidad, por el tono del discurso y, finalmente, por el timbre o materia fónica de los sonidos del poema. En un poema el ritmo es el rasgo distintivo del discurso artístico; así, puede faltar la métrica o la rima, pero no puede faltar el ritmo. Sin el ritmo no hay poesía. En este sentido, decía González Prada, distinguido teórico americano de la ciencia del ritmo — Vallejo lo llamaba «el gran maestro» ${ }^{10}$ - que en poesía: «No poseemos métrica, sino Rítmica» (Exóticas: «Notas») $)^{11}$. Y el mismo Vallejo apuntaba en un artículo publicado en El Comercio, el año de 1929, que: «La poesía es tono [...] lo que importa en un poema [...] es el tono con que se dice una cosa y muy secundariamente lo que se dice». Y por eso concluye que la poesía es intraducible a otro idioma: «Todos sabemos que la poesía es intraducible». Y también que «el tono queda inamovible, en las palabras del idioma original» ${ }^{12}$.

Sostenemos que en la esencia misma de la poesía vallejiana, es decir, en el tono — que él identifica con el ritmo, en general- es por donde tendremos que continuar la búsqueda de la poética o ars de este habilísimo creador. De ahí la importancia de centrar ahora el estudio de su poesía en esta dimensión, es decir, en la aventura del ritmo que significó Trilce. Es aquí en el ritmo donde el poeta se muestra en la inmensidad del poeta castellano que se guarece no solo en el tono - por cierto- sino en la hábil distribución de los golpes acentuales en el verso y en los otros elementos del ritmo que hemos señalado hace un momento. En realidad, desde 1492, el año de Colón, nuestro tatarabuelo el gramático de Nebrija había reparado en la importancia de los golpes acentuales en la poesía castellana.

Solo para proponer un inicio de la búsqueda, miremos estos troqueos perfectos en el poema «II» de Trilce, que emparedan los endeca-

10 Vallejo, César. Poesía completa. Edición, prólogo y notas de Ricardo Silva-Santisteban. Lima, Pontificia Universidad Católica del Perú, 1997, Tomo I, p. 132 y 219.

11 G. Prada, Manuel: Exóticas. Lima, Tipografía de «El Lucero», 1911, p. 156

12 Vallejo, César: «La nueva poesía norteamericana». En: El Comercio, Lima, 30 de julio de 1929. 
sílabos de la estrofa, troqueos y endecasílabos impecablemente disimulados, como corresponde a todo gran artista:

Tiempo Tiempo.

Mediodía estancado entre relentes.

Bomba aburrida del cuartel achica

tiempo tiempo tiempo tiempo.

Este es el esquema acentual (no analizo los dos endecasílabos del medio para no desviarme):

$$
\text { Ó O - Ó O (4) }
$$

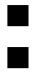

$$
\text { Ó O - Ó O - Ó O - Ó O (8) }
$$

En la estructura pedal del troqueo, como sabemos, la primera sílaba es golpeada por el acento; la otra, no.

Ahora propongo datos concretos de cómo el poeta refilaba los troqueos con fruición. Juan Espejo Asturrizaga, un amigo de Vallejo, en 1965, nos mostró las versiones primigenias de algunos poemas como el que acabo de citar. He visto el facsímil del poema «II», fechado en la cárcel de Trujillo, en 1920, y publicado en la Bobemia de Chiclayo, en 1921, un año antes de la versión definitiva en Trilce. Viene en la edición completa que hizo para la Universidad Católica el distinguido vallejista sanmarquino Ricardo Silva Santisteban ${ }^{13}$. Y es de ver - texto contra texto- cómo el poeta ha pulido y cuadrado el ritmo pedal con extrema sabiduría. Esta es la versión primera, la que fecha en la cárcel:

13 Vallejo, César. Poesía completa. Óp. cit., Tomo II, p. 34. 


\section{Tiempo Tiempo}

Mediodía estancado entre relentes.

Bomba aburrida del cuartel que achica tiempo tiempo.

El esquema muestra la imperfección del verso tercero; igualmente, el recortado troqueo del verso cuarto, que arregla la versión final de 1922.

$$
\text { Ó O - Ó O (4) }
$$

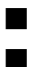

$$
\text { Ó O - Ó O (4) }
$$

Es decir, comparemos —en la versión final, la del libro- cómo en el penúltimo verso ha agregado dos troqueos; y en el verso anterior ha suprimido el feísimo 'que', que malograba la pareja de endecasílabos.

Proponemos, ahora,examinar dos estrofas seguidas de la primera desentonada versión:

Tiempo. Tiempo.

Mediodía estancado entre relentes; bomba aburrida del cuartel, que achica tiempo, tiempo.

$$
\text { Era, era. }
$$

Gallos que cantan escarbando en vano.

Boca del claro día que conjuga:

$$
\text { era, era. }
$$

Y, ahora, escuchemos el ritmo perfecto en las mismas estrofas de la versión final: 


\section{Tiempo Tiempo}

Mediodía estancado entre relentes.

Bomba aburrida del cuartel achica tiempo tiempo tiempo tiempo.

Era Era

Gallos cancionan escarbando en vano.

Boca del claro día que conjuga

era era era era.

El pie trocaico ha hecho lo suyo: la canción versal despliega sus golpes acentuales con silábica belleza musical.

El poeta pudo atreverse con la sintaxis o con el sentido, pero con la simetría del ritmo pedal no; bien sabía que la esencia del verso castellano no permite otra salida: el verso castellano es acentual. El ritmo acentual o de intensidad es el que sostuvo — principalmente — todas las aventuras semánticas o sintácticas en Trilce. Y el poeta fue consciente de los linderos de su quehacer artístico. Así trazó su arte poética, es decir, su poética.

Finalizada ya la epopeya del poemario, en el mismo año en que se propuso cristalizar el gran disparate, aún no repuesto de su fascinante aventura con el lenguaje, Vallejo, el autor, el hombre — poniendo esta vez como testigo al Señor que nos hizo libres-, escribía a su amigo Antenor Orrego lo siguiente, acerca de su libro:

Soy responsable de él. Asumo toda la responsabilidad de su estética. [...] Si no he de ser hoy libre, no lo seré jamás. [...] Me doy en la forma más libre que puedo y esa es mi mayor cosecha artística. [...] iDios sabe hasta dónde es cierta y verdadera mi libertad! [...] iDios sabe cuánto he sufrido para que el ritmo no traspasara esa libertad y cayera en el libertinaje! iDios sabe hasta qué bordes espeluznantes del abismo me he asomado, colmado de miedo... ${ }^{14}$

14 Vallejo, César. Poesía completa.Óp, cit, p. 179. 
Sin duda, debemos agradecer infinitamente el prólogo del mismo Antenor Orrego, que tuvo el privilegio de leer los manuscritos incandescentes del poeta. Un prólogo que infelizmente han suprimido varias reediciones (no la de Silva Santisteban, que aprecio tanto). Ahí Antenor Orrego dejó para la historia la página de aquel que fue el primero en entender el trabajo musical del poeta inmortal; de explicar los límites de su arte poética. Le dice así:

En toda expresión artística hay un quid divinum, un ritmo secreto de entrañada interioridad [...] Las artes todas [...] aspiran, en sus máximas altitudes a la expresión musical. Los grandes creadores solo lo fueron a condición de haber llegado a la música de su arte y de su estilo. Y es que la música es el elemento primario del universo. ${ }^{15}$

Y cierra el prólogo diciéndole así a Vallejo:

Canta tus ritmos divinos, querido; cántalos siempre para que se abracen y se glisen como lianas a mis pensamientos; para que mis lágrimas y mis alegrías y los más escondidos secretos de mi corazón, cuando busquen palabras para incorporarse, encuentren las tuyas, frescas, edénicas y vivas; canta tus himnos para que en la hora en que me suma en el mar de sombra y de callado imperio, me alargues tu mano musical, hermano... ${ }^{16}$

Años más tarde, en un libro que no publicó por propia mano, Vallejo escribía: «iAy del que logra cristalizar un gran disparate!» ${ }^{17}$. Pues bien, el Gran Poeta de Trilce con estos golpes acentuales maestros que le dan ese ritmo inimitable (ese tono, como él decía) a estos versos de un cantar extraño y perfecto logró cristalizar el gran disparate, donde la sintaxis ilógica de la frase y el sentido esquivo se diluyen maravillosamente en el trabajo rítmico de su poesía: con «la música de su arte y de su estilo», como acertó a decirlo su primer gran lector, Antenor Orrego.

15 Ibíd., p. 172

16 Ibíd., p. 178.

17 Vallejo, César: Contra el secreto profesional. Lima: Mosca Azul Editores, 1973, p. 39.

Bol. Acad. peru. leng. 58(58), 2014 


\section{BIBLIOGRAFÍA}

BENDEZÚ, Edmundo. El delirio de los coribantes. (Estudios sobre poética). Lima: P. L. Villanueva, 1981.

McDUFFIE, Keith: "Trilce"I" y la función de la palabra en la poética de César Vallejo». En: Revista Iberoamericana. Pittsburgh, Volumen XXXVI, N. ${ }^{\circ}$ 71, 1970, pp. 191-204.

ORTEGA, Julio: Figuración de la persona. Barcelona: Edhasa, 1971.

VEGAS GARCÍA, Irene: Trilce, estructura de un nuevo lenguaje. Lima: Pontificia Universidad Católica del Perú - Fondo Editorial, 1981.

BALBÍN, Rafael de: Sistema de rítmica castellana. Madrid, Gredos, 1975, $3 .^{\mathrm{a}} \mathrm{ed}$.

VALLEJO, César. Poesía completa. Edición, prólogo y notas de Ricardo Silva-Santisteban. Lima, Pontificia Universidad Católica del Perú, 1997, Tomo I y II.

GONZÁLEZ PRADA, Manuel: Exóticas. Lima, Tipografía de «El Lucero», 1911.

VALLEJO, César: «La nueva poesía norteamericana». En: El Comercio, Lima, 30 de julio de 1929.

VALLEJO, César: Contra el secreto profesional. Lima: Mosca Azul Editores, 1973.

\section{Correspondencia:}

Óscar Coello

Docente del Departamento Académico de Literatura de la Facultad de Letras y Ciencias Humanas de la Universidad Nacional Mayor de San Marcos. Correo electrónico: ocoello@oscarcoello.com 